\title{
Recognition-induced forgetting of faces in visual long-term memory
}

\author{
Kelsi F. Rugo ${ }^{1} \cdot$ Kendall N. Tamler $^{2}$ • Geoffrey F. Woodman ${ }^{2} \cdot$ Ashleigh M. Maxcey $^{3}$
}

Published online: 12 September 2017

(C) The Psychonomic Society, Inc. 2017

\begin{abstract}
Despite more than a century of evidence that longterm memory for pictures and words are different, much of what we know about memory comes from studies using words. Recent research examining visual long-term memory has demonstrated that recognizing an object induces the forgetting of objects from the same category. This recognition-induced forgetting has been shown with a variety of everyday objects. However, unlike everyday objects, faces are objects of expertise. As a result, faces may be immune to recognition-induced forgetting. However, despite excellent memory for such stimuli, we found that faces were susceptible to recognition-induced forgetting. Our findings have implications for how models of human memory account for recognition-induced forgetting as well as represent objects of expertise and consequences for eyewitness testimony and the justice system.
\end{abstract}

Keywords Memory: long-term memory $\cdot$ Face recognition · Visual memory and face recognition $\cdot$ Recognition-induced forgetting $\cdot$ Visual long-term memory

Studies of long-term memory have traditionally used verbal stimuli (e.g., word stem completion tasks; Maxcey, 2016; Palmer, 1999). Yet many everyday long-term memory tasks involve visual, not verbal, stimuli (e.g., Where did I park my

Ashleigh M. Maxcey

ammaxcey@gmail.com

1 Department of Psychology, The University of Utah, Salt Lake City, UT, USA

2 Department of Psychology, Vanderbilt University, Nashville, TN, USA

3 Department of Psychology, The Ohio State University, 271 Psychology Building, 1835 Neil Ave., Columbus, OH 43210, USA car? Do I know that woman? Is that poison ivy?). Research has demonstrated clear differences between long-term memory for pictures relative to words, such as the finding that memory for pictures is almost perfect, unlike memory for words, in laboratory experiments (Standing, 1973).

One implication of near-perfect memory for visual objects stored in long-term memory could be that there is no forgetting of visual stimuli from long-term memory. However, recent work has shown that pictures stored in long-term memory are susceptible to recognition-induced forgetting (Maxcey, 2016; Maxcey \& Bostic, 2015; Maxcey, Bostic, \& Maldonado, 2016; Maxcey, Glenn, \& Stansberry, 2017; Maxcey \& Woodman, 2014). Specifically, practice recognizing some objects held in long-term memory (e.g., a red vase) leads to the forgetting of semantically related objects also held in long-term memory but not accessed (e.g., a blue vase). Although memory for practiced objects would intuitively be higher than memory for objects that were not practiced, memory for these forgotten objects that were semantically related to practiced objects (i.e., related objects) is also lower than memory for nonpracticed objects that were not semantically related to practiced objects (i.e., baseline objects). All studies of this recognition-induced forgetting phenomenon to date have examined the forgetting of everyday objects (e.g., backpacks, donuts, mugs, vases). It is possible that objects of expertise, such as faces, are not vulnerable to recognitioninduced forgetting because of extensive training (Farah, Wilson, Drain, \& Tanaka, 1995, 1998). Here we ask whether faces are vulnerable to recognition-induced forgetting despite people being experts on this class of objects.

Whether faces are susceptible to this type of forgetting is unclear for three main reasons. First, another type of accessbased forgetting, retrieval-induced forgetting (M. C. Anderson, Bjork, \& Bjork, 1994), has examined the forgetting of household items under eyewitness-like situations such as 
sweatshirts and schoolbooks (Shaw, Bjork, \& Handal, 1995), even probing memory for traits such as the actor's hair color (MacLeod, 2002), age or facial hair (Migueles \& GarcíaBajos, 2007), but has never specifically examined memory for one face relative to other faces. Presumably this is because in the retrieval-induced forgetting paradigm, practice typically consists of word stem completion tasks or other verbal tasks, such as cued recall, rather than visual recognition tasks. Therefore it is hard to imagine how one would conduct a retrieval-induced forgetting study with faces. Second, recent evidence demonstrated that temporally grouped objects that belong to a scene (e.g., a cow and a tractor belong to a "farm" scene) do not suffer recognition-induced forgetting, suggesting that not all stimuli that can be semantically grouped together are susceptible to recognition-induced forgetting (Maxcey et al., 2017). Third, a long line of evidence demonstrates that long-term memory for pictures is fundamentally different than memory for words (Calkins, 1898; Durso \& O'Sullivan, 1983; Gehring, Toglia, \& Kimble, 1976; Hockley, 2008; Juola, Taylor, \& Young, 1974; Kirkpatrick, 1894; Madigan, 1974; Münsterberg, 1894; Nelson, Reed, \& McEvoy, 1977; Nelson, Reed, \& Walling, 1976; Paivio \& Csapo, 1973; Paivio, Rogers, \& Smythe, 1968; Paivio, Yuille, \& Madigan, 1968; Snodgrass \& Burns, 1978; Snodgrass, Volvovitz, \& Walfish, 1972; Snodgrass, Wasser, Finkelstein, \& Goldberg, 1974). One way that the processing of visual stimuli differs from verbal stimuli is that visual stimuli are processed across a wider range of expertise. Expertise effects are fundamentally memory effects, because it is what is stored in memory that leads one to be an expert (Chase \& Simon, 1973). However, many studies of expertise effects examine perceptual and attentional differences rather than explicitly testing memory or forgetting (e.g., Reeder, Stein, \& Peelen, 2016; Sunday, McGugin, \& Gauthier, 2017). Therefore, whether objects of expertise, such as faces, are susceptible to recognition-induced forgetting remains unknown.

Faces are a convenient stimulus category of expertise because they allow us to leverage our subjects' lifetime of acquired expertise with faces outside the laboratory with no further training required to achieve expertise status. To create two distinct categories of faces in the present experiment, such that one group is the practiced category (i.e., practiced and semantically related faces), and one is the nonpracticed category (i.e., baseline faces that are neither practiced nor semantically related to practiced faces) similar to previous studies, we used two different races of faces, White and Black. ${ }^{1}$ To eliminate a confounding effect of the relationship between the race of the

\footnotetext{
${ }^{1}$ We do not like the term race because we are all members of the human race, but the field lacks a nomenclature to describe the group membership based on skin color we used in this study. Consistent with the National Institutes of Health nomenclature we use the term race to refer to skin color.
}

subject and the race of the face, which might lead to better recognition of own-race faces (Meissner \& Brigham, 2001), we recruited subjects at a historically Black university and a primarily White institution, where approximately two-thirds of the student population is Black and White, respectively.

In recognition-induced forgetting, the related objects are forgotten relative to baseline objects. Both these classes of objects (i.e., related and baseline) are only seen once during the study phase prior to the memory test phase and neither of them are practiced. The only difference between related and baseline objects is that related objects are from the same category as the practiced objects. Therefore the forgetting of related objects is the consequence of accessing objects, during practice, that are related through their connections in long-term memory.

Here we test two competing hypotheses regarding the forgetting of objects of expertise in the recognition-induced forgetting paradigm. First, experience with faces may lead to denser neural networks representing faces relative to common household objects (Anderson, 1974). If faces are stored in a dense neural network, then the activation of accessing one representation during recognition practice should spread activation to neighboring exemplars. This spread of activation would then require the representation of those activated neighbors to be suppressed so that the target memory could be accessed (Anderson, 2003). If this is the nature of expert memory representations, then we should observe robust recognition-induced forgetting with faces. Second, experience with faces may lead to sharper tuning of the neurons representing faces in the ventral visual stream (Gilbert, Sigman, \& Crist, 2001). If each face is represented by a sharper peak of activation in the network than a common object, then practice recognizing one face would not spread to other faces. This latter hypothesis is consistent with some recent behavioral effects and hypotheses about how the brain represents visual information across repetitions (Bukach, Phillips, \& Gauthier, 2010; Grill-Spector, Henson, \& Martin, 2006). If this second hypothesis is correct, then we should observe no recognition-induced forgetting of faces. As we describe in subsequent sections, our findings support the first of these hypotheses, in which the recognition of one face can result in the forgetting of similar faces.

\section{Method}

\section{Subjects}

A sample of 96 subjects (mean age 21.6 years) is included in all analyses. To determine the necessary sample size, we conducted a pilot experiment with 28 subjects to feed a power analysis (Faul, Erdfelder, Lang, \& Buchner, 2007). In this pilot experiment, the smallest effect we measured had a Cohen's $d=0.68$. If we wanted to have $99 \%$ power to detect 
an effect equal to this with a two-tailed $t$ test, we would require 42 subjects per race group. To ensure adequate power, we collected data from 48 subjects who self-reported being White and 48 Black. Half of the White subjects practiced White faces while the remaining half practiced Black faces. Black subjects were similarly divided such that half of the Black subjects practiced Black faces while the remaining half practiced White faces. Subjects reported normal or correctedto-normal visual acuity and normal color vision. Informed consent was obtained, and all procedures were approved by the Institutional Review Board.

We did not recruit subjects based on race. Therefore we had 27 additional subjects who reported being neither White nor Black participate in the experiment. Because these subjects could not be counterbalanced between same-race and otherrace (i.e., they all belonged to the other-race group since they did not match White or Black), we do not include them in the analyses that examine evidence of group effects. However, we do include all 123 subjects in the analyses below that address the primary question that does not depend on the race of the subject: Are faces immune to recognition-induced forgetting?

\section{Stimuli}

Face stimuli consisted of 60 male faces or 60 female faces (Evans, Marcon, \& Meissner, 2009; Meissner, Brigham, \& Butz, 2005). Half of the faces were Black, and the remaining half were White. The faces were standardized photographs of college age adults wearing a burgundy shirt on a White background facing the camera with a nonsmiling expression (see Fig. 1). Each individual experiment for any given subject only employed either the male faces or female faces. We used stimuli of both genders across subjects to determine whether our results generalize across this variable. The specific roles of all the stimuli (as described below) were counterbalanced across subjects.

\section{Procedure}

The experiment consisted of three phases: the study phase, the practice phase, and the test phase (see Fig. 1). In the study phase, subjects were shown 10 Black and 10 White faces and instructed to remember the faces for a later memory test. Each face was presented for 10 seconds, interleaved by a $500-\mathrm{ms}$

\section{Study Phase}

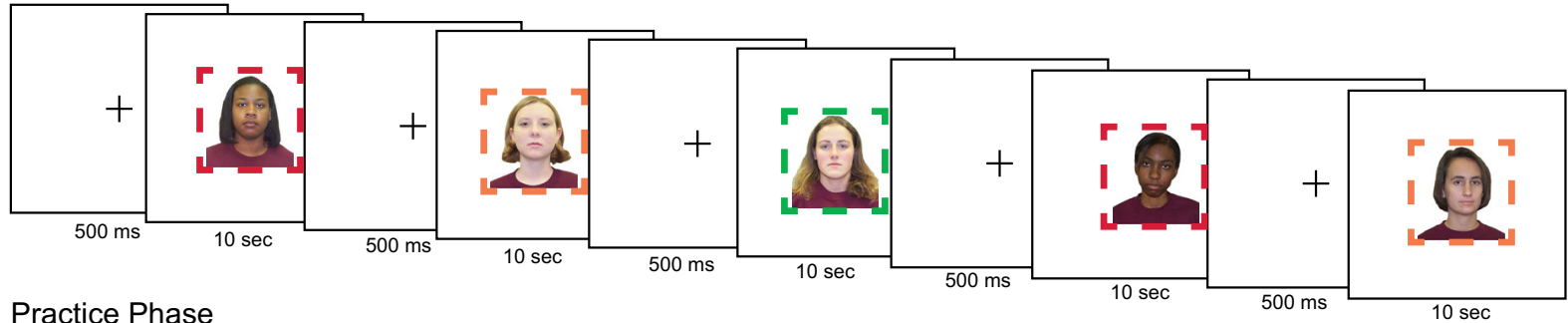

Practice Phase
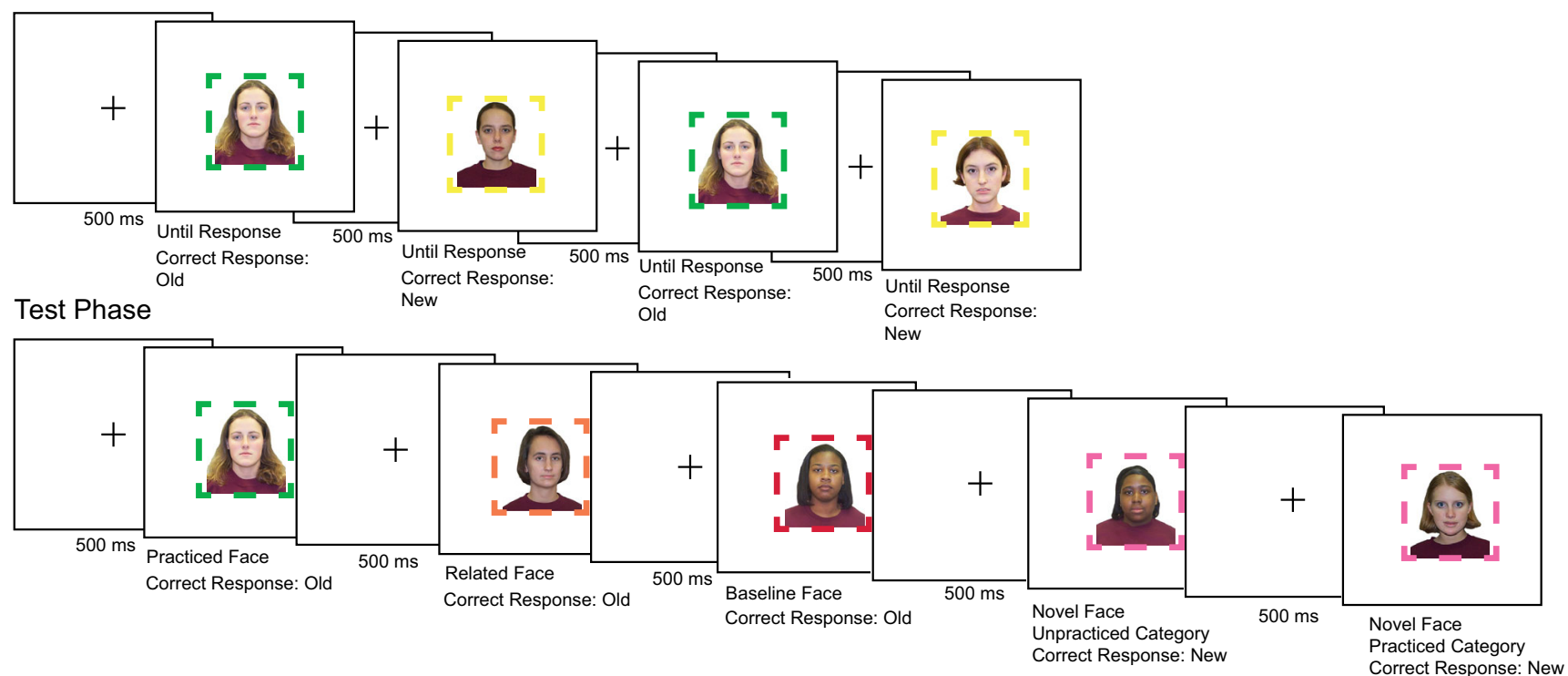

Fig. 1 Stimuli of the experiment. Dashed colored boxes were not presented to the subjects but are used here to illustrate the different face classifications. Faces practiced during the recognition practice phase became the practiced faces and are marked here in green. Novel faces (i.e., practice lures, marked here in yellow) warranted a "new" response and were never again presented in the experiment. Test phase was an oldnew recognition judgment task that presented all the study phase faces and an equal number of novel Black and White faces (marked here in pink). New faces were entirely novel test lures, marked here in pink. (Color figure online) 
Table 1. Hit rates for practiced, related, and baseline faces

\begin{tabular}{lllll}
\hline & & Overall $(N=123)$ & Black $(N=63)$ & White $(N=60)$ \\
\hline Practiced & Hit rate (SE) & $87 \%(.02)$ & $87 \%(.02)$ & $86 \%(.02)$ \\
& A' (B" $\left.{ }_{D}\right)$ & $.91(-.37)$ & $.92(-.41)$ & $.90(-.33)$ \\
Baseline & Hit rate (SE) & $68 \%(.02)$ & $67 \%(.02)$ & $69 \%(.03)$ \\
& A' (B" $\left.{ }_{D}\right)$ & $.84(.34)$ & $.84(.44)$ & $.84(.24)$ \\
Related & Hit rate (SE) & $59 \%(.02)$ & $64 \%(.03)$ & $54 \%(.04)$ \\
& A' (B" $\left.{ }_{\text {D }}\right)$ & $.79(.37)$ & $.82(.27)$ & $.75(.47)$ \\
\hline
\end{tabular}

Hit rates (\% correct, followed by standard error of the mean in parentheses) and A' values (followed by B " ${ }_{D}$ in parentheses) by face classification (i.e., practiced, baseline, and related) collapsed across all 123 subjects in the first column. The second and third columns present the results for the 63 subjects who practiced Black faces and the 60 subjects who practiced White faces, respectively. A 2 (between subjects: practiced Black or White) $\times 2$ (within subjects: baseline B" ${ }_{\mathrm{D}}$ vs. related B" ${ }_{\mathrm{D}}$ ) mixed-model ANOVA demonstrated a reliable interaction, $F(1,120)=11.093, p=.001, \eta_{\mathrm{p}}{ }^{2}=.085$, with a more conservative bias for related White faces and a more liberal bias for related Black faces. There is no reliable difference between related and baseline B" when collapsing across all subjects (i.e., the overall column), $t(121)=.259, p=.796, d=.04$, JZS $=9.66$ in favor of the null, indicating that the significant interaction in $\mathrm{B}{ }_{\mathrm{D}}$ for Black and White faces is an additional demonstration of the polarization between these two conditions.

center fixation cross. The study phase was followed by a 5minute visual distractor task during which subjects searched for Waldo in Where's Waldo? books.

In the second phase, the recognition practice phase, subjects practiced recognizing either half of the Black faces from the study phase or half of the White faces from the study phase. On each trial, one face was presented at center fixation and subjects responded whether the face was "old" by pressing the left key on the response box with their right index finger or indicate that the face was "new" by pressing the second to the leftmost key on the response box with their right middle finger. Each face from the study phase was practiced twice, on two separate trials, such that there were 10 total practice trials to practice the five faces initially shown in the study phase. Randomly interleaved with these studied items were 10 novel faces from the same category (e.g., if Black faces were practiced, the practice lures were also Black), and these served as practice lures requiring a "new" response. The practice phase was followed by an additional 5 minutes of the same visual distractor task that followed the study phase.

The recognition practice phase created three types of faces. Practiced faces were faces that the subject saw during the study phase and practiced during the recognition practice phase. Related faces were the faces that were the same category (i.e., Black or White) as the practiced faces but were not included in the practice phase. Baseline faces were faces from the unpracticed category (e.g., if Black faces were practiced, White faces comprised the category of baseline faces).

In the final test phase, all 20 faces from the study phase were sequentially and randomly presented, interleaved with the same number of novel faces drawn from each category (i.e., 10 novel White faces and 10 novel Black faces). The novel faces from the practice phase used as practice lures were not shown in the test phase. Subjects were asked to indicate whether they had previously seen each face (i.e., an old-new recognition judgment task) and responded in the same fashion as in the practice phase. The novel faces were test lures and the correct response to them was "new."

\section{Data analysis}

The primary dependent variable for recognition memory was hit rate (i.e., hits for practiced, related, and baseline faces), as reported in Table 1 and Fig. 2. We found a similar pattern of results using $A^{\prime}$ (Snodgrass, Levy-Berger, \& Haydon, 1985), reported in Table 1 . We calculate $A^{\prime}$ rather than $d^{\prime}$ because numerous subjects had hit rates of 1.0 for practiced faces, a circumstance under which $d^{\prime}$ is not as reliable as $A^{\prime}$. To provide a way of quantifying the support for the null or alternative hypothesis, we calculated the Scaled JZS Bayes factor for all $t$ tests (as specified in Rouder, Speckman, Sun, Morey, \& Iverson, 2009). Cohen's $d$ measure of effect size also accompanies all significant $t$ tests. In the results below we collapsed across the male and female versions of the experiment because there were no significant differences between the different versions of the experiment, and the pattern of results was the same whether male or female faces were the memoranda.

\section{Results}

We first confirmed that all 123 subjects successfully completed the recognition practice phase (see Table 1, left column). We included the 27 subjects who reported races other than Black and White, in addition to the 96 Black and White subjects, because subject race was irrelevant to this analysis. The grand average performance during the recognition practice phase was well above chance $(84 \%)$, which resulted in reliably better memory for practiced faces $(87 \%)$ relative to baseline faces $(68 \%), t(122)=8.80, p<.001, d=1.08, \mathrm{JZS}=609$, 

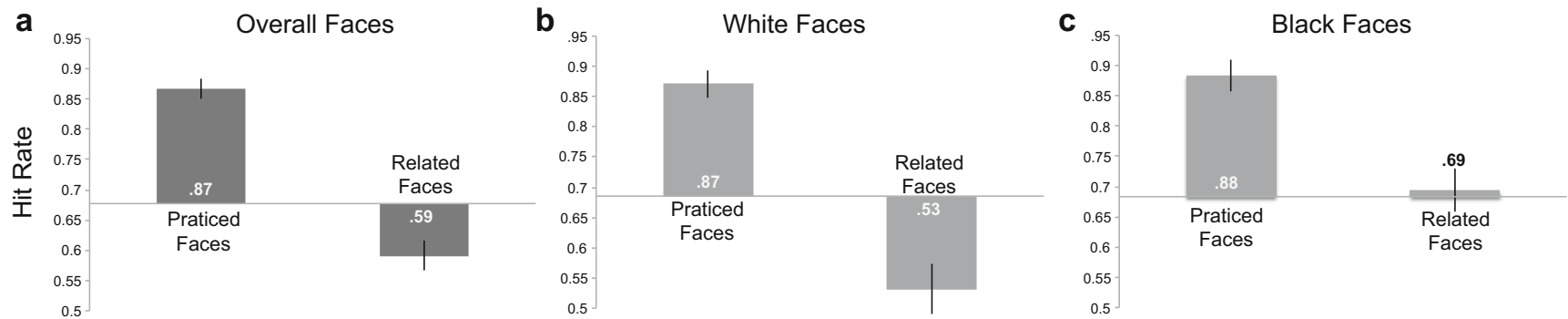

Fig. 2 Hit rates for (a) practiced and related faces in the test phase for all 123 subjects, demonstrating reliably worse memory for related faces compared to baseline faces, (b) the 60 subjects who practiced White faces, showing no evidence of recognition-induced forgetting as memory for related faces was not significantly different than memory for baseline

287, 435, 681 (see Fig. 2a). These 123 subjects demonstrated recognition-induced forgetting, with worse memory for related faces $(59 \%)$ relative to baseline faces $(68 \%), t(122)=3.27$, $p=.001, d=0.38, \mathrm{JZS}=15.10$. This recognition-induced forgetting across all subjects is inconsistent with the general prediction that all faces were immune to recognition-induced forgetting.

Next, we excluded subjects who did not report their race as Black or White (because they cannot contribute to this analysis) and conducted a 2 (between subjects: race of the subject, White or Black) $\times 2$ (between subjects: race of the practiced faces, White or Black) $\times 2$ (within subjects: memory condition, baseline and related ${ }^{2}$ ) mixed-model ANOVA. This analysis led to significant main effects of all three factors, memory condition (baseline and related), $F(1,92)=6.003, p=.016$, $\eta_{\mathrm{p}}{ }^{2}=.061 ;$ race of the subject, $F(1,92)=25.665, p<.001, \eta_{\mathrm{p}}{ }^{2}$ $=.218$; and race of the practiced faces, $F(1,92)=5.871, p=$ $.017, \eta_{\mathrm{p}}{ }^{2}=.060$. There was no interaction between memory condition and race of the subject, $F(1,92)=1.212, p=.274$, $\eta_{\mathrm{p}}{ }^{2}=.013$, or the race of the subject and the race of the practiced faces, $F(1,92)=.120, p=.730, \eta_{\mathrm{p}}{ }^{2}=.001$, and no three-way interaction between memory condition, race of the subject, and race of the practiced faces, $F(1,92)=.284, p=$ $.596, \eta_{\mathrm{p}}{ }^{2}=.003$. There was a significant interaction between memory condition and race of the practiced faces, $F(1,92)=$ $7.869, p=.006, \eta_{\mathrm{p}}{ }^{2}=.079$.

To unpack the significant interaction between memory (baseline and related faces) and race of the practiced faces, we examined recognition-induced forgetting of faces as a function of the race of the practiced faces. Subjects who practiced White faces (see Fig. 2b) showed significant recognition-induced forgetting of related White faces, with superior memory for baseline faces $(69 \%)$ relative to related faces $(53 \%), t(47)=3.437, p=.001, d=$

\footnotetext{
${ }^{2}$ Practiced faces were not included in the ANOVA because the previously reported significant difference between practice and baseline faces would interfere with a true null hypothesis regarding forgetting, as measured by the difference between baseline and related faces.
}

faces, and (c) the 63 subjects who practiced Black faces, demonstrating recognition-induced forgetting in (a) was driven by forgetting of White faces. All $x$-axes represent memory for the baseline faces and error bars represent $95 \%$ confidence intervals as described by Cousineau (2005) with Morey's (2008) correction applied

$.61, \mathrm{JZS}=23.80$. Subjects who practiced Black faces (see Fig. 2c) showed no reliable evidence of recognitioninduced forgetting of related Black faces with similar memory for baseline faces $(68 \%)$ as related faces $(69 \%)$, $t(47)=.277, p=.519, \mathrm{JZS}=6.15$.

We ran this analysis again and included the 27 subjects who reported races other than Black and White because subject race was irrelevant to this analysis (see Table 1, right two columns). Subjects who practiced White faces $(N=60)$ showed significant recognition-induced forgetting of related White faces, with superior memory for baseline faces $(69 \%)$ relative to related faces $(54 \%)$, $t(59)=3.967, p<.001, d=.64, \mathrm{JZS}=115.53$. Subjects who practiced Black faces $(N=63)$ showed no reliable recognition-induced forgetting of related Black faces with similar memory for baseline faces $(67 \%)$ as related faces $(64 \%), t(62)=.648, p=.519, \mathrm{JZS}=5.93$. While we now see a numerical trend in the direction of recognitioninduced forgetting, it is still not reliable despite a sample size larger than that indicated by our power analysis.

An alternative explanation for the immunity of Black faces to recognition-induced forgetting is that at least some of the Black faces are simply more memorable than White faces. This is unlikely for five reasons. First, this stimulus set has previously undergone tests to ensure perceptual ratings of the faces did not significantly differ between White and Black faces (Meissner et al., 2005). Second, memory for baseline faces was $2 \%$ worse for Black than for White faces, $t(121)=.808, p=.034, d=$ $.15, \mathrm{JZS}=3.87$, suggesting that overall White faces were actually more memorable than Black faces. Third, false alarms by subjects who practiced White faces (17.4\%) did not reliably differ from subjects who practiced Black faces $(15.4 \%), t(121)=.862, p=.391, \mathrm{JZS}=3.72$. Fourth, we examined memorability of each individual face stimulus that was employed at test and found that the range of memorability (as measured by baseline memory performance during the test phase) of the Black faces lies within the range of memorability of White faces (see 


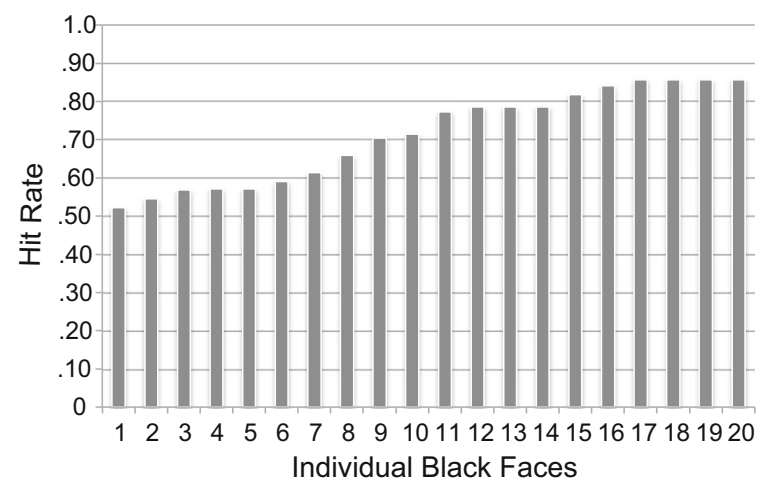

Fig. 3 Item analysis for baseline memory items during the test phase separated by individual stimulus items. Memorability range of Black faces $(52 \%-86 \%)$ was within the range of White faces $(42 \%-100 \%)$,

Fig. 3). Fifth, when equating the range of memorability of White faces with that of Black faces by excluding the six White face stimuli that fell outside the range of baseline memory for Black faces (52\%-86\%), White faces continued to show recognition-induced forgetting, $t(59)=3.45$, $p=.001, d=.48, \mathrm{JZS}=25.84$, whereas Black faces did not.

\section{Conclusion}

Overall, faces were susceptible to recognition-induced forgetting, falsifying the hypothesis that this general class of stimuli are immune to forgetting. These results are consistent with the hypothesis that objects of expertise are stored in dense neural networks that spread activation, consistent with the fan effect (J. R. Anderson, 1974), and that activation of one memory representation spreads to associated nodes.

\section{Discussion}

Here we asked whether recognition-induced forgetting occurs for faces, a convenient stimulus class of expertise. A unique aspect of this study was that the majority of our subjects $(61 \%)$ were non-White university students. This allowed us to control for a confounding effect of the relationship between the race of the stimuli and the race of the observer. We found evidence of recognition-induced forgetting of faces, driven by significant forgetting of White faces. The present results show that objects of expertise such as faces are represented in visual long-term memory similar to everyday objects that are vulnerable to recognition-induced forgetting, such as baskets, vases, and mugs (Maxcey \& Woodman, 2014).

The present findings have two major theoretical implications. First, our observation that accessing one face stored in memory influences the accessibility of related faces stored in memory tells us about the nature of such neural networks.

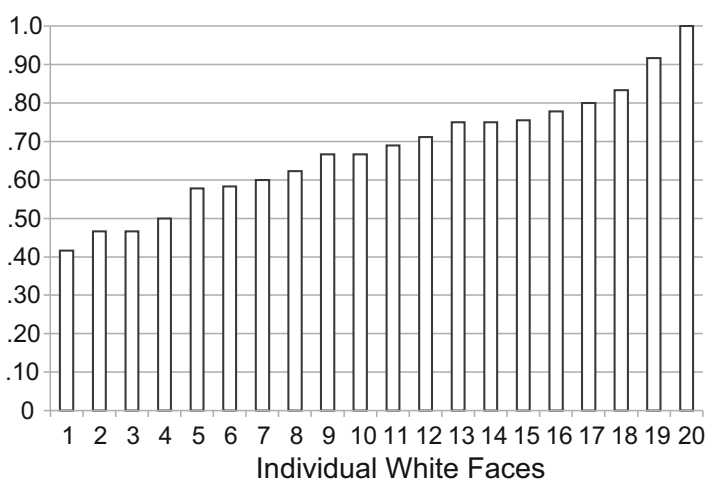

showing that immunity of Black faces to recognition-induced forgetting is not due to a subset of Black faces being particularly memorable

Specifically, the forgetting of faces demonstrates that visual expertise is stored in long-term memory through neural networks that are made increasingly dense with expertise (J. R. Anderson, 1974) instead of representations that are increasingly distinct, in which activation spreads less fluidly with expertise. Second, the present findings illustrate important differences between memory for different race faces. In the present study we did not find reliable evidence for forgetting of Black faces. One potential explanation of this null result is that memory is worse for these faces because there are less exemplars of Black faces stored in memory due to their minority status. This could result in sparser neural network representations of these faces, limiting the spread of activation. Future work could test the hypothesis that ethnic minority faces are immune to recognition-induced forgetting by replicating the present study using stimuli consisting of faces of other ethnic minority groups, such as Asian or Hispanic faces.

Our findings have potential real world consequences such as in eyewitness testimony. Particularly, legal reform has attempted to create a more fair judicial system by incorporating research from cognitive psychology into eyewitness tasks used by law enforcement and in the courtroom (e.g., Wilson, Hugenberg, \& Bernstein, 2013; Wright, Boyd, \& Tredoux, 2001). The present results emphasize the importance of understanding of recognition-induced forgetting in the legal realm. For example, it is possible that recognizing one bank robber from a crime leads to the forgetting of a second bank robber, who subsequently cannot be confidently identified and is not convicted. If ethnic minority faces are indeed immune to this forgetting, this may be an imperative social justice issue because a disproportionate number of racial minorities would be identified relative to White suspects, who are forgotten and go free.

Acknowledgements We thank Christian A. Meissner for the face stimuli. This research was supported by the National Eye Institute of the National Institutes of Health (R01-EY025275, R01-EY019882, R01MH110378, P30-EY08126, and T32-EY007135). 


\section{References}

Anderson, J. R. (1974). Retrieval of propositional information from longterm memory. Cognitive Psychology, 6(4), 451-474.

Anderson, M. C. (2003). Rethinking interference theory: Executive control and the mechanisms of forgetting. Journal of Memory and Language, 49, 415-445. doi:https://doi.org/10.1016/j.jml.2003.08. 006

Anderson, M. C., Bjork, R. A., \& Bjork, E. L. (1994). Remembering can cause forgetting: Retrieval dynamics in long-term memory. Journal of Experimental Psychology: Learning, Memory, and Cognition, 20(5), 1063-1087. doi:https://doi.org/10.1037//0278-7393.20.5. 1063

Bukach, C. M., Phillips, W. S., \& Gauthier, I. (2010). Limits of generalization between categories and implications for theories of category specificity. Attention, Perception, \& Psychophysics, 72(7), 18651874.

Calkins, M. W. (1898). A study of immediate and delayed recall of the concrete and of the verbal. Psychological Review, 5, 451-456.

Chase, W. G., \& Simon, H. A. (1973). Perception in chess. Cognitive Psychology, 4, 55-81.

Cousineau, D. (2005). Confidence intervals in within-subject designs: A simpler solution to Loftus and Masson's method. Tutorial in Quantitative Methods for Psychology,1,42-45.

Durso, F. T., \& O'Sullivan, C. S. (1983). Naming and remembering proper and common nouns and pictures. Journal of Experimental Psychology: Learning, Memory, and Cognition, 9(3), 497-510. doi: https://doi.org/10.1037/0278-7393.9.3.497

Evans, J. R., Marcon, J. L., \& Meissner, C. A. (2009). Cross-racial lineup identification: Assessing the potential benefits of context reinstatement. Psychology, Crime \& Law, 15(1), 19-28.

Farah, M. J., Wilson, K. D., Drain, H. M., \& Tanaka, J. R. (1995). The inverted face inversion effect in prosopagnosia: Evidence for mandatory, face-specific perceptual mechanisms. Vision Research, 35, 2089-2093.

Farah, M. J., Wilson, K. D., Drain, M., \& Tanaka, J. N. (1998). What is "special" about face perception? Psychological Review, 105(3), 482.

Faul, F., Erdfelder, E., Lang, A.-G., \& Buchner, A. (2007). G* Power 3: A flexible statistical power analysis program for the social, behavioral, and biomedical sciences. Behavior Research Methods, 39(2), 175191.

Gehring, R. E., Toglia, M. P., \& Kimble, G. A. (1976). Recognition memory for words and pictures at short and long retention intervals. Memory \& Cognition, 4(3), 256-260. doi:https://doi.org/10.3758/ BF03213172

Gilbert, C. D., Sigman, M., \& Crist, R. E. (2001). The neural basis of perceptual learning. Neuron, 31, 681-697.

Grill-Spector, K., Henson, R., \& Martin, A. (2006). Repetition and the brain: Neural models of stimulus-specific effects. Trends in Cognitive Sciences, 10, 14-23.

Hockley, W. E. (2008). The picture superiority effect in associative recognition. Memory \& Cognition, 36(7), 1351-1359. doi:https://doi. org/10.3758/MC.36.7.1351

Juola, J. F., Taylor, G. A., \& Young, M. E. (1974). Stimulus encoding and decision processes in recognition memory. Journal of Experimental Psychology, 102(6), 1108. doi:https://doi.org/10.1037/h0036370

Kirkpatrick, E. A. (1894). An experimental study of memory. Psychological Review, 1(6), 602.

MacLeod, M. (2002). Retrieval-induced forgetting in eyewitness memory: Forgetting as a consequence of remembering. Applied Cognitive Psychology, 16(2), 135-149. doi:https://doi.org/10.1002/acp.782

Madigan, S. (1974). Representational storage in picture memory. Bulletin of the Psychonomic Society, 4(6), 567-568. doi:https://doi.org/10. 3758/BF03334293
Maxcey, A. M. (2016). Recognition-induced forgetting is not due to category-based set size. Attention, Perception, \& Psychophysics, 78(1), 187-197. doi:https://doi.org/10.3758/s13414-015-1007-1

Maxcey, A. M., \& Bostic, J. (2015). Activating learned exemplars in children impairs memory for related exemplars in visual long-term memory. Visual Cognition, 23(5), 643-558. doi:https://doi.org/10. 1080/13506285.2015.1064052

Maxcey, A. M., Bostic, J., \& Maldonado, T. (2016). Recognition practice results in a generalizable skill in older adults: Decreased intrusion errors to novel objects belonging to practiced categories. Applied Cognitive Psychology doi:https://doi.org/10.1002/acp.3236

Maxcey, A. M., Glenn, H., \& Stansberry, E. (2017). Recognition-induced forgetting does not occur for temporally grouped objects unless they are semantically related. Psychonomic Bulletin \& Review doi:https:// doi.org/10.3758/s13423-017-1302-z

Maxcey, A. M., \& Woodman, G. F. (2014). Forgetting induced by recognition of visual images. Visual Cognition, 22(6), 789-808.

Meissner, C. A., \& Brigham, J. C. (2001). Thirty years of investigating the own-race bias in memory for faces: A meta-analytic review. Psychology, Public Policy, and Law, 7(1), 3.

Meissner, C. A., Brigham, J. C., \& Butz, D. A. (2005). Memory for own - and other - race faces: A dual-process approach. Applied Cognitive Psychology, 19(5), 545-567.

Migueles, M., \& García-Bajos, E. (2007). Selective retrieval and induced forgetting in eyewitness memory. Applied Cognitive Psychology, 21(9), 1157-1172. doi:https://doi.org/10.1002/acp.1323

Morey, R. D. (2008). Confidence intervals from normalized data: A correction to Cousineau (2005). Tutorial in Quantitative Methods for Psychology, 4(2), 61-64. 10.3758/s13414-017-1419-1

Münsterberg, H. (1894). Studies from the Harvard Psychological Laboratory: I. Psychological Review, 1(1), 34.

Nelson, D. L., Reed, V. S., \& McEvoy, C. L. (1977). Learning to order pictures and words: A model of sensory and semantic encoding. Journal of Experimental Psychology: Human Learning and Memory, 3(5), 485. doi:https://doi.org/10.1037/0278-7393.3.5.485

Nelson, D. L., Reed, V. S., \& Walling, J. R. (1976). Pictorial superiority effect. Journal of Experimental Psychology: Human Learning and Memory, 2(5), 523. doi:https://doi.org/10.1037/0278-7393.2.5.523

Paivio, A., \& Csapo, K. (1973). Picture superiority in free recall: Imagery or dual coding? Cognitive Psychology, 5(2), 176-206. doi:https:// doi.org/10.1016/0010-0285(73)90032-7

Paivio, A., Rogers, T. B., \& Smythe, P. C. (1968). Why are pictures easier to recall than words? Psychonomic Science, 11(4), 137-138. doi: https://doi.org/10.3758/BF03331011

Paivio, A., Yuille, J. C., \& Madigan, S. A. (1968). Concreteness, imagery, and meaningfulness values for 925 nouns. Journal of Experimental Psychology Monograph Supplement, 76(1, Part 2), 1-25.

Palmer, S. E. (1999). Vision science: Photons to phenomenology. Cambridge, MA: Bradford Books/MIT Press.

Reeder, R. R., Stein, T., \& Peelen, M. V. (2016). Perceptual expertise improves category detection in natural scenes. Psychonomic Bulletin \& Review, 23(1), 172-179. doi:https://doi.org/10.3758/ s13423-015-0872-x

Rouder, J. N., Speckman, P. L., Sun, D., Morey, R. D., \& Iverson, G. (2009). Bayesian $t$-tests for accepting and rejecting the null hypothesis. Psychonomic Bulletin \& Review, 16, 225-237. doi:https://doi. org/10.3758/PBR.16.2.225

Shaw, J., Bjork, R., \& Handal, A. (1995). Retrieval-induced forgetting in an eyewitness-memory paradigm. Psychonomic Bulletin \& Review, 2(2), 249-253. doi:https://doi.org/10.3758/bf03210965

Snodgrass, J. G., \& Burns, P. M. (1978). The effect of repeated tests on recognition memory for pictures and words. Bulletin of the Psychonomic Society, 11(4), 263-266. doi:https://doi.org/10.3758/ bf03336826

Snodgrass, J. G., Levy-Berger, G., \& Haydon, M. (1985). Human experimental psychology. Oxford, UK: Oxford University Press. 
Snodgrass, J. G., Volvovitz, R., \& Walfish, E. R. (1972). Recognition memory for words, pictures, and words+ pictures. Psychonomic Science, 27(6), 345-347. doi:https://doi.org/10.3758/BF03328986

Snodgrass, J. G., Wasser, B., Finkelstein, M., \& Goldberg, L. B. (1974). On the fate of visual and verbal memory codes for pictures and words: Evidence for a dual coding mechanism in recognition memory. Journal of Verbal Learning and Verbal Behavior, 13(1), 27-37. doi:https://doi.org/10.1016/S0022-5371(74)80027-7

Standing, L. (1973). Learning 10,000 pictures. Quarterly Journal of Experimental Psychology, 25, 207-222. doi:https://doi.org/10. 1080/14640747308400340
Sunday, M., McGugin, R. W., \& Gauthier, I. (2017). Domain-specific reports of visual imagery vividness are not related to perceptual expertise. Behavior Research Methods, 49(2), 733-738. doi: https://doi.org/10.3758/s13428-016-0730-4

Wilson, J. P., Hugenberg, K., \& Bernstein, M. J. (2013). The cross-race effect and eyewitness identification: How to improve recognition and reduce decision errors in eyewitness situations. Social Issues and Policy Review, 7(1), 83-113.

Wright, D. B., Boyd, C. E., \& Tredoux, C. G. (2001). A field study of own-race bias in South Africa and England. Psychology, Public Policy, and Law, 7(1), 119. 